\title{
ARTIGOS
}

\section{BAYLE PROPOSE-T-IL UNE HISTOIRE DE LA PHILOSOPHIE?}

\author{
Hubert Bost* \\ hubert.bost@ephe.sorbonne.fr
}

RESUMO "Propõe Bayle uma história da filosofia ? " A questão não é destinada a receber uma resposta simples. Ela é levantada a fim de interrogar o empreendimento intelectual atípico de Bayle, sua maneira de convocar os filósofos e os sistemas filosóficos no seu pensamento próprio, de os apresentar no seu Dicionário histórico e crítico.

Palavras-chave história da filosofia, história, filosofia, teologia, «Pirro »

RÉSUMÉ "Pierre Bayle propose-t-il une histoire de la philosophie?" La question n'est pas destinée à recevoir une réponse simple. Elle est posée afin d'interroger l'entreprise intellectuelle atypique de Bayle, sa manière de convoquer les philosophes et les systèmes philosophiques dans sa propre pensée, de les présenter dans son Dictionnaire historique et critique.

Mots clés histoire de la philosophie, histoire, philosophie, théologie, "Pyrrhon»

"Pierre Bayle propose-t-il une histoire de la philosophie?" La question, on s'en doute, a quelque chose de rhétorique. Il s'agit moins d'y répondre que

* École Pratique des Hautes Études, Paris. Recebido em 23/07/2009 e aceito em 24/09/2009.

KRITERION, Belo Horizonte, no 120, Dez./2009, p. 295-311. 
de s'en servir pour interroger l'entreprise intellectuelle atypique de Bayle, sa manière de convoquer les philosophes et les systèmes philosophiques dans sa propre pensée, de les présenter dans son Dictionnaire historique et critique.

Qu'entend-on par "histoire de la philosophie"? Je voudrais me poser la question en historien, c'est-à-dire en évitant l'anachronisme. Plusieurs réponses seraient envisageables à partir de la définition courante de cette matière aux $\mathrm{Xx}^{\mathrm{e}}$ et $\mathrm{XXI}^{\mathrm{e}}$ siècles, mais les examiner nous éloignerait de Bayle; on notera simplement qu'une anthologie des grands articles philosophiques du Dictionnaire historique et critique a paru, au début du XXI ${ }^{\mathrm{e}}$ siècle, sous le titre Pierre Bayle. Pour une histoire critique de la philosophie. ${ }^{1}$ Mais qu'entend-on par "histoire de la philosophie" à la fin du XVII" ou au début du XVIII siècle? Il s'agira de déployer cette interrogation selon les catégories et les paradigmes de l'époque à laquelle Bayle a vécu.

À titre liminaire, il faut donc se demander si la question "Pierre Bayle propose-t-il une histoire de la philosophie?" n'est pas anachronique. Le lien qu'elle établit entre l'histoire et la philosophie est-il pertinent? On peut tranquillement écarter cette objection pour deux raisons.

La première est d'ordre biographique: dans sa carrière universitaire, tant à Sedan qu'à Rotterdam, Bayle enseignait la philosophie et l'histoire. Il a donc creusé l'un et l'autre domaine, et, au moins dans sa pratique pédagogique, il a dû réfléchir aux différences et aux ressemblances entre ces deux disciplines, et même à leur possible articulation. Il a pu sentir ce qui les rapprochait - ne serait-ce que négativement, autrement dit par contraste avec la théologie.

Bayle s'est passionné pour l'histoire dès l'époque de sa formation; la lecture de Montaigne lui a appris à réfléchir de façon concrète, en s'appuyant sur les réalités observables et pas seulement en spéculant; Plutarque, dans ses Vie des hommes illustres, avait campé plusieurs personnages de penseurs et avait écrit un traité Sur les opinions des philosophes que Bayle connaît bien. En outre, sa conscience historiographique n'est pas seulement aiguisée par les références au passé, à l'histoire antique: sa correspondance est pleine de considérations sur les événements contemporains, qu'il s'agisse des campagnes militaires ou des courants intellectuels qui s'affrontent; elle atteste

1 Gros, Jean-Michel (éd.). Pierre Bayle. Pour une histoire critique de la philosophie. Choix d'articles philosophiques du Dictionnaire historique et critique. Paris: Honoré Champion, 2001. Le choix de ce titre, qui propose une accroche intéressante, n'est cependant pas commenté dans l'introduction. Les articles retenus sont Chrysippe, Épicure, Leucippe, Manichéens, Origène, Pauliciens, Pascal, Pyrrhon, Rorarius, Spinoza, Zénon d'Élée, Zénon de Sidon, auxquels s'ajoutent le Projet d'un Dictionnaire critique et les Éclaircissements sur les athées, les manichéens et les pyrrhoniens. 
qu'il a très tôt perçu la dimension idéologique du pouvoir politique, que cette idéologie s'adosse sur des croyances religieuses ou rationnelles.

La seconde raison pour laquelle l'établissement d'un lien entre histoire et philosophie ne fait courir aucun risque d'anachronisme se trouve dans les livres de Bayle. Son œuvre philosophique est sans cesse traversée par une interrogation d'historien, dès les Pensées diverses sur la comète (et de manière plus évidente dans la Critique générale de l'histoire du calvinisme de Mr Maimbourg). Le Commentaire philosophique est truffé de références historiques. Inversement, le Dictionnaire, qualifié d'historique et critique, regorge d'articles consacrés à des philosophes - même si, référée à l'ensemble du corpus, la proportion des philosophes peut être jugée modeste. ${ }^{2}$

L'articulation "histoire de la philosophie" - comme, dans un registre différent, le serait "philosophie de l'histoire"3 - est non seulement légitime, mais encore indispensable. En ne considérant chez Bayle que la réflexion philosophique, on ampute son travail et on se prive d'une dimension intellectuelle essentielle - et du reste originale et pertinente - de sa pensée.

2 Rappelons l'estimation d'Elisabeth Labrousse: "Les articles du Dictionnaire consacrés à des philosophes font $5 \%$ du total (104 sur 2044), mais comme plusieurs comptent parmi les plus longs de l'ouvrage, leur étendue constitue un pourcentage sensiblement supérieur de pages. Ces 104 articles se répartissent comme suit: 48 philosophes anciens, 13 médiévaux, 25 auteurs de la Renaissance et 18 auteurs du xvII siècle." Pierre Bayle, II: Hétérodoxie et rigorisme, $2^{e}$ éd. Paris: Albin Michel, 1996. p. 194 n. 32.

3 "On ne sait pas bien où \& comment Chilon, aiant demandé à Esope quelle étoit l'occupation de Jupiter, remporta cette réponse, il abaisse les choses hautes \& éleve les choses basses. Mais on ne peux douter que cette réponse ne soit l'Abrégé de l'histoire humaine. Prenez l'histoire par quelque bout qu'il vous plaira, \& suivez-en les progrès depuis le commencement jusqu'à la fin, vous verrez par-tout des exemples de l'alternative qu'Esope vouloit signifier. Le monde est un véritable jeu de bascule; tour à tour on y monte \& on y descend. On doit admirer dans ce jeu-là les profondeurs d'une sage providence \&l'activité de nos passions. Un homme est-il devenu riche, ses enfans élevez dans l'opulence se remplissent de vanité, sont prodigues \& se ruïnent. Les enfans de ceux-ci, n'aiant pour toute ressource que leur industrie, travaillent nuit \& jour pour s'enrichir, \& s'élevent. Un roiaume aquiert une très-grande puissance, il s'enorgueillit, il traite fiérement ses voisins: chacun craint d'être conquis; \& pour se tirer du danger, on forme des ligues si formidables qu'elles abaissent le prince qui s'étoit tant élevé. Cette régle a ses exceptions, car il y a des familles \& des Etats qui conservent très-long-tems leur élévation. La République romaine, qui abolit tant de souverains, s'augmenta de plus en plus pendant quelques siecles. Les paiens étoient ainsi persuadez que le ciel prenoit à tâche d'humilier les choses hautes qu'ils s'imaginérent des dieux à qui la prospérité des hommes causoit une violente jalousie. Les philosophes mêmes qui nioient la providence reconnoissoient un je ne sai quoi qui affectoit de renverser les grandeurs." "Esope", rem. L. Cette réflexion sur la citation d'Esope, qui fait passer du mythos au logos ou d'une interprétation providentialiste à une lecture immanente de l'histoire, sont d'autant plus intéressants que Bayle a auparavant consacré une remarque aux apologues des fables. Elle se poursuit sur le ton du moraliste, pour se conclure de façon fidéiste: "Si l'homme n'étoit pas un animal indisciplinable, ne se seroit-il pas corrigé de son orgueil, après tant de preuves de la maxime d'Esope réitéréres en chaque païs \& en chaque siecle? D'ici à deux mille ans, si le monde dure autant, les réitérations continuelles de la bascule n'auront rien gagné sur le cœur humain. Pourquoi donc les réitérer sans fin \& sans cesse? II faut mettre le doigt sur la bouche, \& adorer humblement la sagesse du conducteur de cet univers, \& reconnoître en même tems la corruption infinie de notre nature, \& sa servitude sous le joug des impressions machinales, maladie invétérée qui ne céde qu'aux opérations miraculeuses de la grâce." 
Il y a plus: comme philosophe même, Bayle appartient à la génération de la réception de Descartes, c'est-à-dire qu'il hérite en quelque sorte d'une querelle des anciens et des modernes, des aristotéliciens et des cartésiens pour faire court. La discipline que Bayle exerce a certes connu jadis ou naguère bien d'autres mutations, mais celle à laquelle il assiste et participe rappelle à ce penseur attiré par l'histoire l'irréductible pluralité et l'historicité des systèmes philosophiques.

Une raison supplémentaire pousse Bayle à se faire l'historien des systèmes philosophiques: comme il est davantage porté à démonter les systèmes qu'à en forger un lui-même - ce qui est peut-être l'expression de son scepticisme - , il est conduit à les étudier, à les comparer, à les évaluer, mais aussi à les replacer dans leur contexte originel (ne serait-ce que pour les en extraire dans un second temps).

Ces préalables posés, je voudrais m'arrêter d'abord sur l'histoire de la philosophie avant Bayle et de son temps, puis m'interroger sur le rôle de son travail du point de vue de ce qui l'a suivi, et enfin faire une ou deux réflexions inspirées par quelques lignes de l'article "Pyrrhon".

\section{L'histoire de la philosophie avant Bayle et de son temps}

Qui, avant Bayle ou de son temps, fait ce qu'on peut appeller de l'histoire de la philosophie? L'ancêtre du genre est à ma connaissance l'ouvrage latin de Burlaeus sur les Vies des philosophes et des poètes (1477), ${ }^{4}$ que Bayle n'utilise pas; il recourt en revanche souvent au De Scriptoribus Historiae Philosophicae libri IV (1649) de Joannes Jonsius, une synthèse encore presque exclusivement consacrée aux auteurs anciens. ${ }^{5}$ Bréhier avait noté à propos de Jonsius que toute idée de continuité entre Antiquité et Moyen Âge échappe aux penseurs de cette époque, ${ }^{6}$ et que Francis Bacon lui-même concevait encore l'histoire de la philosophie comme 1'histoire des sectes. ${ }^{7}$ À propos d'un autre auteur que Bayle cite souvent, Bréhier établit une comparaison utile pour comprendre

4 Burlaeus, Gualterus [Walter Burley] (1275-1344/45). Libellus de vita et moribus philosophorum et poetarum. Cologne, 1472; Louvain: Jean de Westphalie, in-4; Nuremberg: Frid. Creussener, 1479, in-fol.

5 Jonsius, Joannes (1624-1659). De Scriptoribus historiae philosophicae libri IV. Francofurti: ex off. T. M. Götzii, 1659.

6 Bréhier, Émile. Histoire de la philosophie (1931). Paris: PUF-Quadrige, 1981. t. I, p. 11. II ajoute (ibid.) que de son côté Jean de Launoy (1603-1678), le dénicheur de saints, étudie quelques années plus tard l'histoire des écoles philosophiques médiévales (De Scholis celebrioribus seu a Carolo magno seu post eundem Carolum per Occidentem instauratis liber, 1672).

7 “Qu'on y ajoute les sectes et les controverses les plus célèbres qui ont occupé les doctes; qu'on énumère les auteurs, les livres, les écoles, la suite des chefs d'école, les académies, les sociétés, les collèges, les ordres." (De dignitate et augmentis scientiarum II, IV (1623), cité d’après E. Bréhier, ibid.) 
le paradigme historiographique dans lequel se meut le philosophe, et visà-vis duquel il cherche à prendre ses distances: "Georges Horn, l'auteur de la première histoire générale de la philosophie, qui mène le développement depuis les origines jusqu' au XVIII ${ }^{\mathrm{e}}$ siècle; la préface renvoie à Bacon, et le titre complet de l'ouvrage en indique bien le caractère: Historiae philosophicae libri septem, quibus de origine, successione, sectis et vita philosophorum ab orbe condito ad nostram aetatem agitur. Ce qui l'intéresse, c'est moins l'analyse et la connaissance précise du contenu des doctrines que leur énumération et leur suite; il a, à l'égard de l'histoire de la philosophie proprement dite, la position que l'histoire de l'Église a à l'égard de celle des dogmes; et, pas plus qu'il n'existe à ce moment d'histoire des dogmes, il n'existe une histoire véritable de la philosophie $\gg{ }^{8}$

Croisons les noms des auteurs, prédécesseurs ou contemporains de Bayle, qui ont marqué l'émergence de l'historia philosophica ${ }^{9}$ avec les références bibliographiques qu'on trouve dans le Dictionnaire. ${ }^{10}$ Bayle connaît et cite cinq des sept auteurs de telles histoires (Daniel Morhof, Georgius Hornius, Gerard Vossius, Johann Jonsius, Thomas Stanley, Abraham de Grau et Johannes de Raey): seuls les deux derniers ne sont pas mentionnés. Mais il utilise leurs travaux comme n'importe quel autre ouvrage contenant des renseignements utiles, sans entrer le moins du monde dans un débat de fond avec eux. ${ }^{11}$

8 Hornius, Georgius (1620-1670). Historiae philosophicae libri septem, Leyde, 1655 (voir E. Brehier, ibid., p. 11-12). Bayle cite cet ouvrage en trois occasions anecdotiques: sur l'enseignement du Grec Jean Argyrophile à Rome, sur les honneurs rendus à Aristote après sa mort et sur l'idée de George Hornius selon laquelle Zoroastre était le prophète Balaam (voir resp. "Argyropyle", rem. C, "Aristote" et "Zoroastre"). Deux autres passages où il est question des rapports entre philosophie et théologie ("Hoffman (Daniel)", rem. C, et "Melanchthon", rem. K), seront évoqués plus loin.

9 Je me réfère à la liste établie par Jonathan Israel, "Philosophy, History of Philosophy, and I'Histoire de l'Esprit Humain: A Historiographical Question and Problem for Philosophers", in: J. B. Schneewind (éd.), Teaching New Histories of Philosophy: Proceedings of a Conference. Princeton: University Center for Human Values, 2004. p. 329-344.

10 van Lieshout, Lenie. The Making of Pierre Bayle's Dictionnaire historique et critique, with a CD-ROM containing the Dictionnaire's library and referees between articles. Amsterdam-Utrecht: APA-Holland University Press, 2001.

11 Hornius, Georgius (1620-1670). Historiae philosophicae libri VII, quibus de origine, successione, sectis et vita philosophorum ab orbe condito ad nostram aetatem agitur (1655): Argyropyle, rem. C; Aristote (philos.), rem. S; Hoffman, rem. C; Melanchthon, rem. K; Zoroastre. - Jonsius, Joannes (1624-1659). De Scriptoribus historiae philosophicae libri IV (1659): Ammonius (divers); Andronicus (philos.), rem. C; Apulée, rem. A; Arcesilas, rem. D; Aristarque (gramm.), rem. A; Ariston, rem. G; Aristote (philos.), rem. B, C; Aristote (arch.), rem. A, B; Artemidore, rem. C; Asclepiade (de Pruse), rem. F; G; Carneade, rem. E, F, N, P; Chrysippe (fils), rem. C, D; Chrysippe (philos.), rem. C; Craterus, rem. C; Cratippe, rem. C; Demetrius, rem. A, B; Democrite, rem. D; Dicearque (disc.), rem. A; Diogene (stoïc.), rem. C; Ephore, rem. B, E; Epicure, rem. D; Hierocles (philos.), rem. C; Junon, rem. E; Lucrece (poète), rem. M; Theopompe, rem. A. K; Zenon (d'Elée), rem. C; Zenon (de Sidon), rem. A, B. - Morhof, Daniel Georg (1639-1691). Polyhistor, 1688: Adam (M.); Allatius, rem. F; Andrelinus; Aprosio (A.), rem. A; Barleus (G.), rem. F; Barthius, rem. O; Baudius, rem. D; Boccalin, rem. F; Bonciarius; Bongars, rem. B, I; Brunus, rem. C; Erasme, rem. CC; Grotius (H.), rem. G; Kuhlman; Lingelsheim, rem. B; Lotichius (P. neveu), rem. G, H; Macedo (F.); Melanchthon, rem. K; Pythagoras, rem. L; Puteanus, rem. F; Ruarus, rem. B; Sacratus, rem. A; Sadeur; Scioppius, rem. R; Spinosa (J. de), rem. 
Significativement, lorsqu'il parle du célèbre traité de Francis Bacon, Bayle note que "le traité de Augmentis Scientiarum, qui fut réimprimé à Paris l'an 1624, est une des meilleures productions de l'auteur', mais il ne s'arrête pas au contenu de l'ouvrage et n'en souligne pas l'importance pour la définition de la philosophie. Il est plus intéressé par l'histoire de la réception, et par l'idée que lui-même se fait de l'utilisation possible d'un tel Dictionnaire:

Voici ce que Costar en écrivoit à Voiture: "J'ai lu depuis quelques mois le livre que le Chancelier Bacon a fait du Progrès des Sciences, où j'ai trouvé beaucoup de choses admirables ». Il rapporte ensuite quelques-unes de ces choses, et fait voir par ce choix-là son bon goût; car, en effet, ce sont toutes belles \& grandes pensées. J'ai ouï dire que les Euvres de Bacon étoient un des livres que Costar manioit le plus, \& qu'il en tiroit le fond ou la base de ses recueils: c'est-à-dire qu'aiant trouvé des les écrits de Bacon quelque pensée qui lui plaisoit, il l'écrivoit sur une feuille; \& puis, quand il rencontroit dans d'autres livres quelque chose qui se rapportoit à cela, il l'ajoutoit à cette feuille, après quoi, il ne manquoit pas de répertoire ni de lieux communs. ${ }^{12}$

La comparaison du travail de Bayle avec celui de ses prédécesseurs ou de ses contemporains montre évident que son approche est très différente, à la fois beaucoup moins systématique et beaucoup plus personnelle que celle des "historiens de la philosophie". Son utilisation des travaux d'historiens de la philosophie est très partielle, il y glane les renseignements dont il juge avoir besoin mais n'envisage pas du tout de les imiter. En quoi il ressemble davantage à un polygraphe érudit et curieux comme Pierre Costar (1603-1660) qu'à un historien soucieux d'exhaustivité. L'absence d'entrées consacrées à Platon ou à Descartes, par exemple - alors qu'Aristote et Spinoza ${ }^{13}$ ont chacun un long article - montre que Bayle ne nourrit pas l'ambition de couvrir l'ensemble de la discipline.

Là où, en revanche, Bayle se montre davantage "historien de la philosophie" que ses prédécesseurs ou contemporains, c'est dans la conscience qu'il a de l'évolution et de la spécificité de cette discipline par rapport à la théologie;

B; Wouwer, rem. B, D, G. -Stanley, Thomas (1625-1678). Historia Philosophiae orientalis (tr. Le Clerc) (1690): Adam, rem. K; Zoroastre, rem. A, B, D. - Vossius, Gerardus Ioannus (1577-1649). De Philosophia et Philosophorum Sectis Libri II (1658): Actuarius, rem. A; Adam, rem. G; Ammonius (divers); Anaxagoras, rem. K; Andromaque (de Crète); Andronicus (philos.), rem. D; Averroes, rem. A, D, H; Barbarus (H.), rem. G; Carneade, rem. B; Chrysippe (philos.), rem. U; Criton (divers), rem. A; Diogene (stoïc.), rem. E; Melampus, rem. G; Pyrrhon, rem. A, C; Pythagoras, rem. B, E; Tyrannion (gramm.), rem. D.

12 "Bacon, François", rem. B. Bayle mentionne ce traité dans les articles "Aristote" (intéressant sur la réception d'Aristote, notamment à cause de son influence en théologie) et "Machiavel", rem. E (comme Abraham de Wicquefort, Bacon avait noté que Machiavel dit ce que font les princes, non ce qu'ils devraient faire).

13 On sait que Bayle fait jouer à Spinoza un rôle idéal-typique dans l'histoire de la philosophie - J.-M. Gros parle d'"un spinozisme construit sur mesure" (ibid., p. 513) - qui le conduit à se méprendre sur l'originalité du philosophe d'Amsterdam. 
ou, pour le dire dans des termes plus conflictuels, ce cette obligation dans laquelle elle se trouve de toujours et maintenir son autonomie par rapport à cette théologie dont la pente naturelle est de chercher à la soumettre ou à l'instrumentaliser. Cet enjeu est crucial dans les questions où la construction de la notion de Dieu par la métaphysique affronte la révélation chrétienne. S'appuyant sur Hornius, Bayle parle de cette tension entre philosophie et théologie à propos de la question de la double vérité: Jacob Thomasius examine dans l'un de ses discours "si une chose peut être vraie en philosophie $\&$ fausse en théologie, comme quelques-uns l'ont prétendu; \& il observe qu'entre ceux qui ont osé soutenir un tel paradoxe, les uns ont été poussez par un respect excessif pour Aristote, \& les autres par une haine déréglée pour ce philosophe. Ceux-ci étoient les théologiens." 14 Bayle prolonge la réflexion:

Thomasius a raison de dire que ce fut une chose très-scandaleuse de voir soutenir
qu'il est vrai en philosophie que Dieu est auteur du péché par accident, mais que cela
n'est pas vrai en théologie. [...] un tel partage de la vérité est un moien de fournir
les erreurs les plus impies: car en effet rien ne plus propre que cela à introduire le
pyrrhonisme, puisqu'en raisonnant de la sorte on réduit la vérité à la condition des
qualitez corporelles. De ce que le même corps nous paroît petit ou grand selon que
nous le voïons ou sans lunettes ou avec des lunettes, on a droit de conclure que nous
ignorons qu'il est grand ou s'il est petit absolument parlant, \& que la petitesse ou
la grandeur absolue des corps nous est inconnue. Si donc la même proposition était
vraie \& fausse selon qu'on la considéreroit ou en théologien ou en philosophe, il
s'ensuivroit nécessairement que nous ne connoîtrions pas la verité en elle-même, \&
qu'elle ne consisteroit que dans un rapport muable aux dispositions de notre esprit,
comme la bonté des viandes ne consiste que dans un certain rapport aux dispositions
de la langue, lesquelles venant à changer, sont cause que les aliments qui étoient bons
ne le sont plus.

Hornius permet encore à Bayle de rappeler que Daniel Hoffman (15401617), un théologien luthérien qui avait violemment combattu l'enseignement de la philosophie dans les Académies, considérait cette discipline comme "trèspernicieuse \& selon laquelle plusieurs véritez théologiques étoient fausses". Il obtint dans un premier temps que ses opposants soient déchus du ministère pastoral, mais l'autorité du prince apaisa la dispute, et "il falut qu'Hoffman calât les voiles". ${ }^{15}$ Je risque une interprétation de cette anecdote: un prince

14 "Hoffman (Daniel), rem. C. Le philosophe Jacob Thomasius (1622-1684) - qui doit, au xvıII siècle, une partie de sa notoriété au fait d'avoir été le professeur de Leibniz - est souvent cité par Bayle. Parmi les théologiens qui ne supportaient pas Aristote, il convient de ne pas compter Mélanchthon: "C'est à tort que quelques-uns l'ont accusé de haïr la philosophie péripatéticienne”, affirme Bayle ("Melanchthon”, rem. K) contre un amalgame du père Rapin qui a mal lu Hornius.

15 "Hoffman (Daniel)", rem. C. Ce bref portrait d'un théologien buté et hostile à la philosophie appartient à la riche collection des allusions polémiques voilées de Bayle contre Pierre Jurieu. 
sage contient les velléités plus ou moins fanatiques des théologiens et permet à la raison et à la rationalité d'exercer leurs fonctions; au fond, il existe sur le plan intellectuel une situation analogue à celle dont on est plus familier sur le plan sociopolitique, quand Bayle parle de la nécessité d'encadrer les religions pour leur interdire de s'emparer du pouvoir.

Avant de faire l'hypothèse d'une écriture hypocrite de sa part de Bayle à propos de ce qu'il dit sur la doctrine de la double vérité, il convient de bien préciser les données du problème: il s'agit pour lui $1^{\circ}$ de ne rien céder sur les droits de la philosophie à raisonner et à spéculer; $2^{\circ}$ de prouver à ses éventuels détracteurs que la liberté du philosophe ne menace pas la révélation parce qu'elle se déploie dans un champ différent; $3^{\circ}$ et donc de montrer qu'elle n'oppose en aucune façon une vérité d'un certain ordre à une vérité d'un autre ordre $^{16}$. Si, d'un côté, Bayle se dit convaincu de l'incapacité de la raison à rendre compte de certains mystères - dans ces protestations de fidéisme que beaucoup de commentateurs considèrent comme des grimaces, d'un autre côté il se bat farouchement pour conserver au philosophe (et à l'historien) la plus grande latitude dans ses recherches et son expression.

C'est ce qu'illustre un passage d'une remarque de l'article "Luther" où Bayle défend la spécificité de la philosophie en donnant des gages aux examinateurs sourcilleux sur l'orthodoxie. Luther, disent ses détracteurs, ne comprenait pas la théologie scolastique, "il enseignoit qu'un même dogme est faux \& vrai en même tems, faux en philosophie, vrai en théologie”. Bayle s'efforce de calmer le jeu:

Disons [...] qu'il se peut mêler du mal-entendu dans cette dispute-là, \& beaucoup de logomachies, \& qu'on blâmeroit à tort la doctrine de Luther s'il l'eût exprimée de cette façon: les mêmes dogmes qui paroissent faux \& impossibles quand on n'en juge que par les lumieres naturelles sont vrais \& certains quand on en juge par les lumieres de la parole de Dieu. Mais de prétendre qu'après même que la Révélation nous a fait connoître qu'une doctrine est véritable, elle continue d'être fausse en philosophie, c'est s'abuser. Il est bien plus juste de reconnoître que les lumieres philosophiques, dont l'évidence nous avoit paru un guide certain pour juger des choses, étoient trompeuses \& illusoires, \& qu'il les faut rectifier par les nouvelles connoissances que la révélation nous communique. ${ }^{17}$

16 Les Éclaircissements sur les athées, les manichéens et les pyrrhoniens montrent clairement que Bayle revendique fermement sa liberté de penser et son devoir critique; mais il s'adresse à une instance théologico-ecclésiastique (le consistoire de l'Église wallonne de Rotterdam): il s'efforce de prouver à ses membres que, en application de leurs propres principes, ils ne sauraient s'effaroucher de son éventuelle audace: Jésus, Paul, les Pères et les théologiens ont toujours considéré que la vérité révélée n'avait pas de compte à rendre à une raison humaine trop infirme pour accéder à ses mystères.

17 "Luther (Martin)", rem. KK. 
On peut certes trouver qu'il y a là bien des contorsions, que Bayle n'est pas sincère, qu'il se dissimule. Mais si l'on interprète sa remarque comme une tentative pour expliquer à des non philosophes que la philosophie se donne un cadre épistémologique qu'elle n'ambitionne pas de dépasser, on peut en faire une lecture bien différente: l'histoire de la philosophie étudie l'évolution d'une discipline qui a pris conscience, face aux autres disciplines et notamment à la théologie, qu'elle doit viser une vérité "dans les limites de la simple raison" et s'interdire d'aller au-delà. Ce qui semble une courbette devant la révélation apparaît alors, paradoxalement, comme l'expression du sentiment de supériorité de celui qui connaît et maîtrise son champ de compétences.

Lorsqu'il raconte la vie des philosophes, Bayle ne fait guère montre d'originalité sinon par sa rigueur critique dans l'établissement des faits ou la chronologie, et parfois par son humour. L'approche biographique lui permet de présenter les systèmes de pensée non comme des doctrines abstraites, mais comme des lieux de débat, souvent sources de conflit, parfois de souffrance. Mais ce qui frappe dans la façon qu'il a d'exposer ces systèmes, c'est son implication. Loin de s'abstraire comme le ferait aujourd'hui l'auteur d'un article de dictionnaire, Bayle intervient, pousse les raisonnements, imagine ce qu'aurait dû dire ou répliquer tel protagoniste, cherche des solutions. C'est souvent le cas dans de grands articles du Dictionnaire tels que "Manichéens" ou "Spinoza", et il serait intéressant de relever les interventions rédactionnelles où il montre qu'il en a conscience. C'est aussi le cas lorsque, se substituant à Straton de Lampsaque ou prolongeant ce qu'il considère comme son système, il élabore un "stratonisme" qui lui doit son existence même. ${ }^{18}$

Bayle recourt si fréquemment à des procédés littéraires qu'à l'évidence il veut conjurer le risque d'ennui que pourrait susciter un article purement historico-philosophique: ainsi il fait intervenir des modernes dans des articles consacrés à des penseurs de l'Antiquité, ${ }^{19}$ se sert du stoïcien Chrysippe comme caution de discussions chrétiennes et modernes sur la liberté de l'homme et toute-puissance de Dieu, ${ }^{20}$ ou encore écrase délibérément la chronologie afin de

18 Continuation des Pensées diverses 112: OD III, p. 343a.

19 Exemples de ces sauts chronologiques: l'intervention de Malebranche sur la providence et la nature des choses ("Chrysippe", rem. T), celle d'Arnauld sur le plaisir et le bonheur (“Epicure”, rem. H; voir aussi Du Rondel à la rem. L, Gassendi à la rem. M).

20 "Chrysippe", rem. H, où s'invite le calvinisme. Voir Jacqueline Lagrée, "La critique du stoïcisme dans le Dictionnaire de Bayle", in: De l'humanisme aux Lumières. Bayle et le protestantisme. Mélanges E. Labrousse, Paris-Oxford: Universitas-Voltaire Foundation, 1996, p. 581-593. "Il y a une certaine désinvolture dans la présentation de la théorie stoïcienne par Bayle”, remarque J.-M. Gros, présentation de l'article "Chrysippe", dans Pierre Bayle. Pour une histoire critique de la philosophie, p. 73. 
dégager des invariants, des correspondances entre anciens et modernes. ${ }^{21}$ Ici il opère des sauts géographiques, ${ }^{22}$ là il invente des fictions: le débat imaginaire d'Épicure et un platonicien, puis avec prêtre païen, ${ }^{23}$ le dialogue de Melissus le partisan de l'unité de Dieu et Zoroastre le dualiste et les apologues un peu scabreux de la mère laissant sa fille partir au bal destiné à faire apparaître les implications de la question manichéenne, ${ }^{24}$ et bien sûr l'intervention de l'abbé pyrrhonien. ${ }^{25}$

\section{Remarques en contrepoint sur la "vraie" histoire de la philosophie}

Envisageons à présent 1'histoire de la philosophie au moment où elle émerge véritablement dans sa spécificité. Après l'Historia philosophiae de Thomas Stanley $(1711)^{26}$ et l'Histoire critique de la philosophie de BoureauDeslandes, ${ }^{27}$ 1'Historia critica philosophiae de Johann Jacob Brucker ${ }^{28}$ est considérée comme la première véritable histoire de la philosophie; ${ }^{29}$ Jacques Proust a montré qu'elle constituait un chaînon intermédiaire entre les deux grands projets alphabétiques que sont le Dictionnaire de Bayle et l'Encyclopédie de Diderot. ${ }^{30}$

Que nous apprennent les innombrables histoires de la philosophies qui se sont multipliées à partir du milieu du XVIII ${ }^{\mathrm{e}}$ siècle? D'abord ceci: en rigueur de termes, l'histoire de la philosophie, c'est de l'histoire; or, dans les faits, ce sont des philosophes qui l'écrivent (ou des historiens des idées

21 Le cartésianisme à propos de Leucippe (“Leucippe”, rem. B). Voir aussi Spinoza, qualifié d'”athée de systême, \& d'une méthode toute nouvelle, quoi que le fond de sa doctrine lui fût commun avec plusieurs philosophes anciens \& modernes, Européens \& Orientaux" (Spinoza”, rem. A). Voir Guido Canzani, "Les philosophes de la Renaissance italienne dans le Dictionnaire", in: H. Bots (éd.), Critique, savoir et érudition à la veille des Lumières. Le Dictionnaire de Pierre Bayle (1647-1706), Amsterdam-Maarssen, APA, 1998, p. 143ss.

22 Voir "Spinoza", rem. B et X; "Zabarella”, rem. G, où le débat sur la preuve de l'existence de Dieu passe de l'aristotélisme à l'athéisme chinois.

23 "Epicure", rem. S et T.

24 "Manichéens", rem. D; "Pauliciens", rem. F.

25 "Pyrrhon", rem. B.

26 Thomas Stanley (1625-1678), Historia philosophiae vitas, opiniones resque gestas et dicta philosophorum sectae cujusvis complexa, Leipzig: Thomam Fritsch, 1711, 2 vol. in-4.

27 André-François Boureau-Deslandes (1690-1757), Histoire critique de la philosophie, Amsterdam: F. Changuion, 1737, 4 vol. in-12.

28 Jacob Brucker (1696-1770), Historia critica philosophiae a mundi incunabulis ad nostram usque aetatem deducta, $1^{\text {re }}$ édition Leipzig, 1741-1744 et 1767, 6 vol. in-4.

29 Jacques Proust, Diderot et l'Encyclopédie, rééd. Paris: Albin Michel, 1995, p. 244.

30 Id., ibid., p. 244-254; voir aussi Pierre Rétat, Le Dictionnaire de Bayle et la lutte philosophique au xVIII siècle, Paris: Les Belles Lettres, 1971, p. 389-392 et 394, notamment n. 164), qui souligne a contrario la rupture qu'introduit Brucker en critiquant Bayle que son scepticisme relègue à l'écart de la philosophie de son temps. Cependant P. Rétat abonde dans le sens de J. Proust en expliquant (p. 391) que Diderot dispose, avec Brucker, d'une synthèse pratique de tout ce dont il aurait besoin chez Bayle. 
ou des historiens de la pensée que les "vrais" historiens ne reconnaissent pas toujours comme leurs pairs). ${ }^{31}$ L'histoire de la philosophie est pratiquée par des philosophes qui s'intéressent d'une part à l'évolution historique de leur discipline (axe diachronique), d'autre part au rapport entre les systèmes et les sociétés dans lesquelles ils naissent (axe synchronique). Pour une raison simple: il faut une vaste culture philosophique pour comprendre les problématiques de ces penseurs, et a priori seuls les chercheurs de la discipline ont des chances d'en saisir les enjeux. (On pourrait faire une remarque analogue pour l'histoire du droit, de la médecine et de la théologie, pour s'en tenir aux trois grandes disciplines universitaires médiévales.) Mais renversons la question, et interrogeons-nous un instant sur ce que serait une histoire de la philosophie pratiquée par un "vrai" historien. On y retrouverait évidemment toute la question de l'interface entre la société et la pensée, celle des paradigmes culturels qui régissent une époque ou une aire géographique, de la doxa et des bouleversements qu'elle subit. Mais n'y rencontrerait-on pas aussi l'histoire des philosophes, une approche biographique que l'histoire de la philosophie a progressivement évacuée ou du moins considérablement réduite: la vie des philosophes, leurs brouilles, les misères, les mesquineries; mais aussi une interrogation sur le statut social du penseur, son mode de vie, ses revenus, sa légitimité sociale: toutes choses qu'il semble évident aujourd'hui de rencontrer en histoire depuis que Jacques Le Goff a écrit Les intellectuels au Moyen Âge $e^{32}$ ou en sociologie lorsqu' on lit Homo academicus de Pierre Bourdieu, ${ }^{33}$ mais qu'il n'est guère fréquent de croiser en histoire de la philosophie. Pour de bonnes raisons: les sources sont rares, les anecdotes sujettes à caution, etc. Néanmoins, autant on peut constater que Bayle rompt avec ce qui précède et que, s'il influence fortement les générations qui le suivent, ses lecteurs historiens de la philosophie ne sont pas ses disciples, autant l'on peut souligner que Bayle avait pressenti la pertinence ou l'intérêt d'une approche qui aurait, plus que nous ne le pratiquons nous-mêmes aujourd'hui, entrelacé la question historique et la question philosophique. N'est-ce pas là une clé herméneutique, à laquelle chacun recourt plus ou moins consciemment lors de ses consultations du Dictionnaire? Le corps de l'article donne les faits historiques et amorce la discussion philosophique que développent les remarques. Bayle distingue les approches méthodologiques

31 Pour une réflexion sur ce point, voir "Historians Look at the New Histories of Philosophy", table ronde avec Lorraine Daston, Anthony Grafton, Jonathan Israel et Donald R. Kelley, Princeton University, 7 avril 2003.

32 Jacques Le Goff, Les intellectuels au Moyen Âge, Paris: Seuil, 1957, $2^{\mathrm{e}}$ éd. 1985.

33 Pierre Bourdieu, Homo academicus, Paris: Minuit, 1984. 
des deux disciplines, mais entrelace volontiers leurs problématiques dans son écriture. C'est bien pourquoi ni l'historien ni le philosophe ne sont satisfaits: le premier voit la chronologie violée, le second s'impatiente de lire le rappel de détails insignifiants. Mais ce procédé, qui paraîtra un peu "fourrre-tout" une ou deux générations plus tard, ${ }^{34}$ correspond à l'épistémè et à l'esthétique de son temps. Il y a du baroque dans l'écriture de Bayle car il faut, pour le plaisir du lecteur, comme un compositeur de musique, multiplier les thèmes et les motifs, les faire jouer l'un avec l'autre, surprendre, relancer l'attention. A ce titre, Bayle propose une approche historique de la philosophie qui ne peut pas devenir un système, qui n'a pas vocation à s'imposer comme méthode, mais qui offre la trajectoire rare et singulière d'un lecteur érudit et curieux, virtuoso tant historien savant que philosophe redouté. ${ }^{35}$

\section{La mise en abyme de l'histoire de la philosophie: "Pyrrhon"}

Bien des arguments convergent donc vers une conclusion négative: Bayle ne propose, et n'a jamais eu l'intention de proposer une histoire de la philosophie. D'ailleurs, l'ordre alphabétique n'est pas le plus propice à ce type d'exercice. Il n'en reste pas moins que son écriture est en permanence traversée par une conscience historique. Conscience que non seulement les systèmes de pensée doivent être replacés dans le contexte de l'époque où ils sont nés sans toutefois les réduire à de simples expressions de de ce contexte -, mais que c'est aussi vrai de la façon de philosopher, de l'idée que l'on se fait de cette activité. Le constat de l'historien croise et nourrit le projet du philosophe:

Notez que l'Antiquité avoit deux sortes de philosophes. Les uns ressembloient aux avocats, \& les autres aux rapporteurs d'un procès. Ceux-là en prouvant leurs opinions, cachoient autant qu'ils pouvoient l'endroit foible de leur cause \& l'endroit fort de leurs adversaires. Ceux-ci, savoir les sceptiques ou les académiciens, représentoient fidélement \& sans nulle partialité le fort \& le foible des deux partis opposez. ${ }^{36}$

34 On connaît la remarque de Voltaire dans Le Temple du goût (1731): "Tout l'esprit de Bayle se trouve dans un seul tome, de son propre aveu; car ce judicieux philosophe, ce juge éclairé de tant d'auteurs et de tant de sectes, disait souvent qu'il n'aurait pas composé plus d'un in-folio s'il n'avait écrit que pour lui et non pour les libraires."

35 Michelet peut être considéré, à certains égards, comme un héritier de cette tradition qui refuse d'avoir à choisir: "Nous nous occuperons à la fois de l'étude de l'homme individuel, et ce sera la philosophie - et de l'étude de l'homme social, et ce sera l'histoire." (Cours de l'École Normale, 1829, cité par G. Monod, La Vie et la Pensée de Jules Michelet (1798-1852), Paris: Champion, 1923, 2 vol., t. I, p. 127, cité par Marc Bloch en note dans Apologie pour l'histoire ou métier d'historien, Paris: A. Colin, 1949, rééd. 1993).

36 "Chrysippe", rem. G. 
Bayle considère en effet que la bonne façon de pratiquer la philosophie correspond à la seconde possibilité. La suite du passage montre que Bayle est convaincu de la supériorité d'une méthode qui assume le doute, l'hésitation, la nuance. Du reste, il déplore l'incapacité de quelques philosophes et des théologiens en général à prendre du recul:

Cette distinction a été vue fort peu parmi les chrétiens dans les écoles de philosophie,
$\&$ encore moins dans les écoles de théologie. La religion ne souffre pas l'esprit
académicien; elle veut qu'on nie ou que l'on affirme. On n'y trouve point de
juges qui ne soient parties en même tems: on y trouve une infinité d'auteurs qui
plaident la cause selon la maxime de Chrysippe, je veux dire qui se tiennent dans
la simple fonction d'avocat; mais on n'y trouve presque point de rapporteurs: car
si quelqu'un représente de bonne foi \& sans nul déguisement toute la force du parti
contraire, il se rend odieux \& suspect, \& il court risque d'être traité comme un infâme
prévaricateur.

Si la discipline philosophique passe par la mise en question des évidences, l'un de ses moteurs est nécessairement le doute: on doit douter pour se mettre à penser. Il faut certes distinguer le doute sceptique (pyrrhonien) et le doute méthodique (cartésien), mais l'un et l'autre prennent appui sur cette posture intellectuelle du refus de prendre la doxa, les évidences, pour argent comptant. La notion de scepticisme recouvre certes une école philosophique spécifique, mais elle renvoie, plus universellement, au geste philosophique lui-même. C'est par exemple le cas lorsque, dans la remarque B de l'article "Pyrrhon", Bayle soutient que le cartésianisme a donné au scepticisme classique sa véritable envergure:

A peine connoissoit-on dans nos Ecoles le nom de Sextus Empiricus; les moiens de l'époque (=époquè) qu'il a proposez si subtilement n'y étoient pas moins inconnus que la Terre Australe lorsque Gassendi en a donné un abrégé qui nous a ouvert les yeux. Le cartésianisme a mis la derniere main à l'œuvre; \& personne parmi les bons philosophes ne doute plus que les sceptiques n'aient raison de soutenir que les qualitez des corps qui frappent nos sens ne sont que des apparences.

Bien qu'il ne porte que sur la question de la perception, ce petit résumé montre Bayle à la fois conscient du caractère historique de la pensée philosophique, et cependant convaincu qu'il y a, au bout du compte, une certaine proximité entre les intuitions de Sextus et celles des cartésiens: comme historien des idées, il est attentif à l'évolution, aux effets de rupture, aux déplacements des problématiques, bref: à la diachronie; et comme philosophe, il perçoit les permanences, les homologies par dela les différences d'époque: en un mot, la synchronie. 
C'est surtout dans la fermeté de la distinction entre philosophie et théologie qu'éclate cette conscience de la spécificité de la première. Le doute est le moteur de la philosophie comme la foi est celui de la théologie: "Il n'y a [...] que la religion qui ait à craindre le pyrrhonisme: elle doit être appuiée sur la certitude; son but, ses effets, ses usages, tombent dès que la ferme persuasion de ses véritez est effacée de l'ame." Cherchant à rassurer - ou à endormir - les gardiens de l'orthodoxie religieuse, Bayle ajoute aussitôt une précision censée montrer qu'il n'empiète pas sur leur territoire; cette précision elle-même, drapée dans les atours de la plus pure doctrine, n'est pourtant pas sans arrière-pensée:

Mais d'ailleurs on a sujet de se tirer d'inquiétude; il n'y a jamais eu, \& il n'y aura jamais qu'un petit nombre de gens qui soient capables d'etre trompez par les raisons des sceptiques. La grâce de Dieu dans les fidelles, la force de l'éducation dans les autres hommes, \& si vous voulez même, l'ignorance \& le panchant naturel à décider, font un bouclier impénétrable aux traits des pyrrhoniens, quoique cette secte s'imagine qu'elle est aujourd'hui plus redoutable qu'elle n'étoit anciennement.

Il n'y a guère d'exemple où l'indécision, ou l'incapacité de la raison à trancher, éclate avec plus de netteté que lorsque la raison affronte le problème du mal ("Manichéens", "Pauliciens"). Si l'approche de Bayle ulcère le consistoire de l'Église wallonne de Rotterdam, ${ }^{37}$ c'est parce qu'elle lui semble empiéter sur le domaine réservé de la théologie et de l'Église: toute question métaphysique s'interrogeant sur Dieu à partir de la raison alerte les dogmaticiens, et toute relecture de l'histoire des hérésies fait lever le sourcil aux historiens ecclésiastiques. Mais le soupçon des chiens de garde de l'orthodoxie est-il fondé? Plus exactement, doit-on aujourd'hui rester prisonniers des cadres dans lesquels les acteurs du temps envisageaient leurs désaccords? Pour l'historien, cette attitude serait une démission: donner tort ou raison à tel ou tel protagoniste, c'est renoncer à rendre compte des enjeux du conflit. Juger n'est pas le travail de l'historien. Il est bien plus intéressant de chercher à comprendre comment Bayle se situe dans le champ du débat. Or la tactique de Bayle, entre évitement et affrontement, s'explique par la perception qu'il a des enjeux proprement philosophiques du débat. Proprement philosophiques même s'ils sont débattus dans un cadre ecclésial, ou en recourant à un vocabulaire religieux; proprement philosophiques parce qu'il s'agit de Dieu envisagé à

37 Voir "L'Affaire Bayle". La bataille entre Pierre Bayle et Pierre Jurieu devant le consistoire de l'Église wallonne de Rotterdam. Texte établi et annoté par H. Bost. Introduction par A. McKenna, Saint-Étienne: Institut Claude Longeon, 2006. 
partir des seules capacités (ou des incapacités) humaines. L'aporie à laquelle aboutit la confrontation des raisons a priori (l'unité de Dieu) défendues par Melissus et des raisons a posteriori (rendre compte des phénomènes, à savoir la coexistence du bien et du mal) invoquées par Zoroastre dans "Manichéens" montre par exemple que Bayle choisit une configuration historique précise afin d'en dégager une problématique philosophique universelle en passant par une reformulation originale et personnelle: le manichéisme au sein du christianisme lui fournit un exemple, mais il ne s'agit pas pour lui de décider qui a tort ou raison. L'historien Bayle fournit le matériau dont le philosophe Bayle dégage une sorte d'idéal-type.

On comprend qu'avec l'approche qu'il défend dans la remarque B de "Pyrrhon", une théologie rationnelle apparaisse à Bayle comme un objet assez monstrueux. Mais si l'on postule que le scepticisme correspond chez lui à une attitude critique fondamentale, tant en histoire qu'en philosophie; qu'il constitue une condition de possibilité nécessaire au discours scientifique, on mesure l'intérêt de cette histoire fragmentaire de la philosophie qu'il écrit dans le Dictionnaire: le scepticisme élitiste hérité du libertinage érudit de La Mothe le Vayer, qui transparaît encore quelque peu ici dans la condescendance à l'égard d'esprits peu capables de penser, est dépassé, ${ }^{38}$ voire sublimé, lorsqu'il n'est plus un objectif mais un moyen: Bayle est bien moins concerné par cette recherche d'une sagesse supérieure que par la mise en place d'un protocole rigoureux destiné à émanciper définitivement la philosophie de la théologie. Or l'histoire est ici une alliée de la philosophie: ces deux disciplines ne peuvent se déployer qu'à condition d'être débarrassées des contrôles doctrinaux, des examens d'orthodoxie. L'histoire, pour établir les faits et en proposer une interprétation, doit neutraliser les efforts que les Églises de tous bords déploient pour en faire, selon l'expression de Melchior Cano, un lieu théologique; la philosophie, pour penser les concepts et en explorer les potentialités, doit s'affranchir des efforts des dogmaticiens pour consacrer, ou du moins pour contrôler, son cheminement. Bien qu'elles aient des épistémologies et des méthodologies différentes, histoire et philosophie s'appuient sur la seule raison et s'efforcent d'écarter ce qui est susceptible d'en empêcher le déploiement. Il n'y a d'histoire possible qu'à partir du présupposé suivant: Dieu ne dicte pas l'histoire des hommes (ou s'il la dicte, ce n'est pas vers lui que l'attention doit se porter). Il n'y a de philosophie envisageable que dans le renoncement

38 Voir Ruth Whelan, "The wisdom of Simonides: Bayle and La Mothe le Vayer", in: R. Popkin - A. Vanderjagt (éd.), Scepticism and Irreligion in the Seventeenth and Eighteenth Centuries, Leyde: Brill, p. 230-253; Frédéric Brahami, Le travail du scepticisme. Montaigne, Bayle, Hume, Paris: PUF, 2001. 
à toute autorité inatteignable par les capacités rationnelles et spéculatives de l'homme. L'histoire de la philosophie ne peut être celle d'une vérité qui se dévoilerait progressivement: elle n'est que celle des efforts accomplis par les hommes pour se comprendre, comprendre le monde et l'histoire.

\section{Références bibliographiques}

BAYLE, Pierre. Dictionnaire historique et critique. ["cinquième" édition], AmsterdamLeyde-La Haye-Utrecht, 1740, 4 vol. in fol.

Bayle, Pierre, Eeuvres diverses. La Haye, 1727-1731, 4 vol. in fol.

"L'Affaire Bayle". La bataille entre Pierre Bayle et Pierre Jurieu devant le consistoire de l'Église wallonne de Rotterdam. Texte établi et annoté par H. Bost. Introduction par A. McKenna. Saint-Étienne: Institut Claude Longeon, 2006.

Bloch, Marc. Apologie pour l'histoire ou métier d'historien. Paris: A. Colin, 1949, rééd. 1993.

Bourdieu, Pierre. Homo academicus. Paris: Minuit, 1984.

Boureau-Deslandes, André-François. Histoire critique de la philosophie. Amsterdam: F. Changuion, 1737, 4 vol. in-12.

Brahami, Frédéric. Le travail du scepticisme. Montaigne, Bayle, Hume. Paris: PUF, 2001.

BRÉHIER, Émile. Histoire de la philosophie (1931). Paris: PUF-Quadrige, 1981.

BRUCKER, Jacob. Historia critica philosophiae a mundi incunabulis ad nostram usque aetatem deducta. $1^{\text {re }}$ édition Leipzig, 1741-1744 et 1767, 6 vol. in-4.

Burlaeus, Gualterus [Walter Burley]. Libellus de vita et moribus philosophorum et poetarum. Cologne, 1472; Louvain: Jean de Westphalie,?, in-4; Nuremberg: Frid. Creussener, 1479, in-fol.

CAnzani, Guido. "Les philosophes de la Renaissance italienne dans le Dictionnaire", in: H. Bots (éd.), Critique, savoir et érudition à la veille des Lumières. Le Dictionnaire de Pierre Bayle (1647-1706), Amsterdam-Maarssen, APA, 1998, p. 143-164.

Gros, Jean-Michel (éd.). Pierre Bayle. Pour une histoire critique de la philosophie. Choix d'articles philosophiques du Dictionnaire historique et critique. Paris: Honoré Champion, 2001.

Hornius, Georgius. Historiae philosophicae libri septem. Leyde, 1655.

Israel, Jonathan. "Philosophy, History of Philosophy, and l'Histoire de l'Esprit Humain: A Historiographical Question and Problem for Philosophers", in: J. B. Schneewind (éd.), Teaching New Histories of Philosophy: Proceedings of a Conference. Princeton: University Center for Human Values, 2004, p. 329-344.

Jonsius, Joannes. De Scriptoribus historiae philosophicae libri IV. Francofurti: ex off. T. M. Götzii, 1659.

Labrousse, Elisabeth. Pierre Bayle, II: Hétérodoxie et rigorisme. $2^{\mathrm{e}}$ éd. Paris: Albin Michel, 1996. 
LAgRÉE, Jacqueline. "La critique du stoïcisme dans le Dictionnaire de Bayle", in: De l'humanisme aux Lumières. Bayle et le protestantisme. Mélanges E. Labrousse. ParisOxford: Universitas-Voltaire Foundation, 1996, p. 581-593.

Le Goff, Jacques. Les intellectuels au Moyen Âge. Paris: Seuil, 1957, 2éd. 1985. Monod, Gabriel. La Vie et la Pensée de Jules Michelet (1798-1852). Paris: Champion, 1923.

Proust, Jacques. Diderot et l'Encyclopédie. rééd. Paris: Albin Michel, 1995.

RÉtAT, Pierre. Le Dictionnaire de Bayle et la lutte philosophique au XVIII siècle. Paris: Les Belles Lettres, 1971.

Stanley, Thomas. Historia philosophiae vitas, opiniones resque gestas et dicta philosophorum sectae cujusvis complexa. Leipzig: Thomam Fritsch, 1711, 2 vol. in-4. VAN LiEshout, Lenie. The Making of Pierre Bayle's Dictionnaire historique et critique, with a CD-ROM containing the Dictionnaire's library and referees between articles. Amsterdam-Utrecht: APA-Holland University Press, 2001.

Voltaire. Le Temple du goût (1731).

Whelan, Ruth. "The wisdom of Simonides: Bayle and La Mothe le Vayer", in: R. Popkin - A. Vanderjagt (éd.), Scepticism and Irreligion in the Seventeenth and Eighteenth Centuries. Leyde: Brill, p. 230-253. 